\title{
Continuum mechanics of media with inner structures
}

\author{
Anna Duyunova \\ V. A. Trapeznikov Institute \\ of Control Sciences \\ of Russian Academy of Sciences, \\ Moscow, Russia \\ anna.duyunova@yahoo.com \\ Valentin Lychagin \\ V. A. Trapeznikov Institute \\ of Control Sciences \\ of Russian Academy of Sciences, \\ Moscow, Russia \\ University of Troms $\varnothing$, Norway \\ valentin.lychagin@uit.no \\ Sergey Tychkov \\ V. A. Trapeznikov Institute \\ of Control Sciences \\ of Russian Academy of Sciences, \\ Moscow, Russia \\ sergey.lab06@ya.ru
}

May 13, 2020

\begin{abstract}
We propose a geometrical approach to the mechanics of continuous media equipped with inner structures and give the basic (mass conservation, Navier-Stokes and energy conservation) equations of their motion.
\end{abstract}




\section{Introduction}

The classical continuum mechanics deals with media in three-dimensional spaces and describes them by a system of partial differential equations on a 3D manifold $M$. Usually, $M$ is a domain in $\mathbb{R}^{3}$ or $\mathbb{R}^{+} \times \mathbb{S}^{2}$. In such cases, elements of the medium are points. The idea that the medium has inner structure leads to a more interesting picture.

Let us consider, for example, a diatomic gas. A molecule of such gas is composed of two atoms. We have a homonuclear gas if its molecule is composed of two atoms of the same element, e.g. oxygen $\mathrm{O}_{2}$, or a heteronuclear gas if it is composed of two atoms of different elements, like carbon monoxide CO. Thus, for description of such gases, we use $M=\mathbb{R}^{3} \times \mathbb{R} P^{2}$ for the homonuclear gases and $M=\mathbb{R}^{3} \times \mathbb{S}^{2}$ for heteronuclear ones. In the case of atmospheric gases, the configuration manifold $M$ is the total space of the bundle $\pi: M \rightarrow \mathbb{R}^{+} \times \mathbb{S}^{2}$, with fibers diffeomorphic to $\mathbb{R} \mathbb{P}^{2}$ or $\mathbb{S}^{2}$, depending on the type of the gas. Here the projection $\pi$ indicates the mass center of molecule. The case of water, $\mathrm{H}_{2} \mathrm{O}$, is more interesting, here the configuration space of inner states is the circle bundle of the tangent space to a two-dimensional sphere, and this space is diffeomorphic to $\mathbb{R P}^{3}$ (see, for example, [5]). An example of a medium composed with solids, i. e. the bundle $\pi: \mathbb{R}^{3} \times S O(3) \rightarrow \mathbb{R}^{3}$ is trivial, were given by Cosserat brothers [12], for a modern discussion see [11].

These examples give motivation for the following definition of a medium with inner structure used in this paper.

Namely, by a configuration space of such medium we mean:

1. A smooth bundle $\pi: M \rightarrow B$, where $M$ and $B$ are Riemannian manifolds equipped with metrics $g_{M}$ and $g_{B}$ respectively.

2. In order to compare inner structures (fibers of $\pi$ ) at different points of $B$, we assume that the bundle $\pi$ is equipped with a connection $\nabla_{\pi}$. This connection splits tangent spaces $T_{m} M$ into the vertical $T_{m}^{v} M$ and horizontal $H_{m} \stackrel{\pi_{*}}{\simeq} T_{\pi(m)} B$ parts, i. e. $T_{m} M=T_{m}^{v} M \oplus H_{m}$, where $T_{m}^{v} M$ and $H_{m}$ are $g_{M}$-orthogonal, and the restriction of the metric $g_{M}$ on $H_{m}$ coincides with $g_{B}$. Moreover, we require that the parallel transport of fibers along the connection $\nabla_{\pi}$ be an isometry of fibers with respect to the metric induced by $g_{M}$.

3. Flow in the medium is given by a $\pi$-projectable vector field $X$ on $M$, i. e., $X$ preserves the bundle $\pi$. This field can be split due to the connection $\nabla_{\pi}$ into the sum $X=X_{H}+X_{V}$, where $X_{V}$ is a $\pi$-vertical 
field and $X_{H}$ is a horizontal lift of the vector field $X^{\prime}=\pi_{*}(X)$ on the base manifold $B$.

The paper is organized as follows. First of all, we discuss the thermodynamics of media based on the measurement [7] of internal energy and deformation tensor. Especially, we discuss Newtonian media that have $O\left(g_{M}\right)$ in a general case and $O\left(g_{B}\right) \times O\left(g_{F}\right)$ symmetries, when media have an inner structure. Using the Rosenlicht and Procesi theorems (see [9], [6],[10]), we find the fields of rational invariants, and describe state equations for internal energy and stress tensor in their terms. We formulate the thermodynamic state equations in terms of Lagrangian manifolds, additionally equipped with a Riemannian structure. This approach introduces these manifolds as an intrinsic part of the continuum mechanics, as well as it allows us to describe different critical phenomena in a pure geometrical manner (see, for example, [3], [4], 2], [8]). The second part of the paper contains basic equations for media: the mass conservation, Navier-Stokes and energy conservation [1]. We outline shortly the coordinateless method of getting these equations, which makes them more transparent (at least from our point of view).

\section{Thermodynamics of media with inner struc- ture}

The thermodynamics is based on measurement [7] of two extensive quantities: the internal energy density $\varepsilon$ and the rate of deformation $\Delta=d_{\nabla} X \in$ End $T$, where $\nabla$ is the Levi-Civita connection associated with the metric $g_{M}$, and $d_{\nabla}$ is the covariant differential. The corresponding dual, or intensive, quantities are the temperature $\theta$ and the stress tensor $\sigma \in \operatorname{End} T^{*}$. We will use the duality of End $T$ and End $T^{*}$ given by the pairing $\langle\sigma, \Delta\rangle=\operatorname{Tr} \sigma \Delta^{*}$.

Thus, the thermodynamic phase space of the medium is

$$
\Phi=\mathbb{R}^{3} \times \operatorname{End} T^{*} \times \operatorname{End} T
$$

with elements

$$
(s, \theta, \varepsilon, \sigma, \Delta)
$$

where $s$ is the entropy density.

In order to write down the first law of thermodynamics, we adopt the following construction.

Let $(\mathcal{A}, \star)$ be an $\mathbb{R}$-algebra. Consider the algebra $\mathcal{A} \otimes$ End $T$ (in this section $\left.\otimes_{\mathbb{R}}=\otimes\right)$ with the multiplication given by $(a \otimes A)(b \otimes B)=(a \star b) \otimes$ $(A B)$. On this algebra, we define a mapping called trace $\operatorname{Tr}_{\mathcal{A}}: a \otimes A \mapsto a \operatorname{Tr} A$. 
In the same way we define the algebra $\mathcal{A} \otimes \operatorname{End} T^{*}$. Then we define $\star$-pairing $\left(\mathcal{A} \otimes\right.$ End $\left.T^{*}\right) \times(\mathcal{A} \otimes$ End $T) \rightarrow \mathcal{A}$ as follows

$$
(a \otimes A) \star(b \otimes B)=(a \star b) \operatorname{Tr}_{\mathcal{A}} A^{*} B, \quad \text { where } \quad A \in \operatorname{End} T^{*}, B \in \operatorname{End} T \text {. }
$$

Further, the cases of algebras $\mathcal{A}$ of exterior $(\star=\wedge)$ and symmetric $(\star=\cdot)$ forms will be used.

The phase space $\Phi$ is a contact manifold equipped with the structure form [7]

$$
\alpha=d s-\theta^{-1} d \varepsilon+\theta^{-1} \sigma \cdot d \Delta .
$$

The first law of thermodynamics means that the thermodynamic state is a maximal integral manifold of this differential form, i. e. a Legendrian manifold $L \subset(\Phi, \alpha)$ of dimension $\operatorname{dim}(\operatorname{End} T)+1$.

Because the entropy is not involved in the conservation laws that govern medium motion, we eliminate it from our geometrical description of the thermodynamics. To this end, we consider the projection $\phi$ of the contact manifold $\Phi$ on the symplectic manifold $(\widetilde{\Phi}, d \alpha)$, where $\widetilde{\Phi}=\mathbb{R}^{2} \times \operatorname{End} T^{*} \times \operatorname{End} T$ and $\phi(s, \theta, \varepsilon, \sigma, \Delta)=(\theta, \varepsilon, \sigma, \Delta)$.

Then the restriction of the mapping $\phi$ on the Legendrian manifold $L$ is a local diffeomorphism on the image $\widetilde{L}=\phi(L)$, and, therefore, $\widetilde{L} \subset \widetilde{\Phi}$ is an immersed Lagrangian manifold in a $2(\operatorname{dim}(\operatorname{End} T)+1)$-dimensional symplectic manifold equipped with the structure form

$$
d \alpha=\theta^{-2}(d \theta \wedge d \varepsilon+\theta d \sigma \wedge d \Delta+\sigma d \Delta \wedge d \theta) .
$$

Thus, the first law of thermodynamics can be reformulated by saying that the thermodynamic state is a Lagrangian submanifold of the symplectic manifold $(\widetilde{\Phi}, d \alpha)$.

Given a Lagrangian manifold $\widetilde{L}$ the corresponding Legendrian manifold $L$ can be reconstructed up to the translation along $s$-axis. It fits to our understanding of entropy as a function defined up to a constant.

In addition, we also require (see [7] for more details) that the quadratic differential form

$$
\kappa=\theta^{-2}(d \theta \cdot d \varepsilon+\theta d \sigma \cdot d \Delta+\sigma \cdot d \Delta \cdot d \theta)
$$

be negative-definite on the Lagrangian submanifold $\widetilde{L}$. The submanifold $\Sigma \subset \widetilde{L}$, where quadratic form $\kappa$ is degenerated, divides $\widetilde{L}$ into domains, where $\kappa$ is negative definite, and the rest. The domains, where $\kappa$ is negative definite, correspond to different phases of the medium, all other domains are domains, where the thermodynamic state is unstable. 
The symplectic structure defines the Poisson bracket on functions on $\widetilde{\Phi}$ of the form

$$
[F, G]=\frac{\theta}{2}\left(\frac{\partial G}{\partial \Delta} \cdot \frac{\partial G}{\partial \sigma}-\frac{\partial F}{\partial \Delta} \cdot \frac{\partial G}{\partial \sigma}+\theta\left(\frac{\partial G}{\partial \varepsilon} \frac{\partial F}{\partial \theta}-\frac{\partial G}{\partial \varepsilon} \frac{\partial G}{\partial \theta}\right)-\sigma \cdot\left(\frac{\partial F}{\partial \varepsilon} \frac{\partial G}{\partial \sigma}-\frac{\partial G}{\partial \varepsilon} \frac{\partial G}{\partial \sigma}\right)\right) .
$$

Hence, the thermodynamic state of the medium can be also defined by equations

$$
F_{k}(\theta, \varepsilon, \sigma, \Delta)=0, \quad k=1, \ldots, \operatorname{dim}(\operatorname{End} T)+1,
$$

where $\left[F_{k}, F_{l}\right]=0$ due to the system (1).

For example, if we have coordinates $(\varepsilon, \Delta)$ on $\widetilde{L}$, then the Legendrian manifold $L$ may be written in the form

$$
s=f(\varepsilon, \Delta), \quad \theta^{-1}=f_{\varepsilon}, \quad \sigma=\left(f_{\varepsilon}\right)^{-1} f_{\Delta} .
$$

Another presentation that we'll use in this paper is given by the coordinates $(\theta, \Delta)$ on $\widetilde{L}$. Then

$$
s=s(\theta, \Delta), \quad \sigma=\sigma(\theta, \Delta), \quad \varepsilon=\varepsilon(\theta, \Delta)
$$

on $\widetilde{L}$ the condition gives us

$$
\left.\alpha\right|_{\widetilde{L}}=\left(s_{\theta}-\theta^{-1} \varepsilon_{\theta}\right) d \theta+\left(s_{\Delta}-\theta^{-1} \varepsilon_{\Delta}+\theta^{-1} \sigma\right) d \Delta=0,
$$

or

$$
\varepsilon_{\theta}=\theta s_{\theta}, \quad \varepsilon_{\Delta}=\theta s_{\Delta}+\sigma .
$$

Let us introduce the density of Helmholtz free energy $h=h(\theta, \Delta)$ on $L$, $\underset{\widetilde{L}}{h}=\varepsilon-\theta s$, then we get the following equations for the Lagrangian manifold $\widetilde{L}$

$$
\sigma=h_{\Delta}, \quad \varepsilon=(\theta h)_{\theta} .
$$

The quadratic differential form $\kappa$ is of the form

$$
\kappa=-\sum_{i, j, k, l} f_{\Delta_{i j} \Delta_{k l}} d \Delta_{i j} \otimes d \Delta_{k l}+2 f_{\varepsilon \Delta} \cdot d \varepsilon \cdot d \Delta-f_{\varepsilon \varepsilon} d \varepsilon^{2}
$$

in the first case, and

$$
\kappa=\theta^{-2}\left(\left(2 h_{\theta}+\theta h_{\theta \theta}\right) d \theta^{2}+2\left(h_{\Delta}+\theta h_{\Delta \theta}\right) d \theta d \Delta+\theta \sum_{i, j, k, l} h_{\Delta_{i j} \Delta_{k l}} d \Delta_{i j} \otimes d \Delta_{k l}\right)
$$

in the second one. 


\section{Thermodynamic invariants of Newtonian media}

Let us consider a medium that possesses a symmetry given by an algebraic group $G \subset G L(T)$. Then this $G$-action on the tangent space $T$ can be prolonged to the contact $G$-action on thermodynamic phase space $\Phi$, if we assume that this action is trivial on $\mathbb{R}^{3}=(s, \theta, \varepsilon)$ and natural on $\operatorname{End} T^{*} \times$ End $T$.

Let $J_{1}, \ldots, J_{N}$ be a set of algebraically independent rational $G$-invariants on $\Phi$, which generate the field of rational $G$-invariants and, therefore, separate regular $G$-orbits (Rosenlicht theorem [9]).

Then a regular $G$-invariant thermodynamic state, i. e. a $G$-invariant algebraic Legendrian manifold $L \subset \Phi$ such that almost all $G$-orbits in $L$ are regular, can be written in the form of $s=f\left(J_{1}, \ldots, J_{N}\right)$ in the case (2), or $h=h\left(J_{1}, \ldots, J_{N}\right)$ in the case (3)), where $f$ and $h$ are rational functions.

In this section, we consider Newtonian media, i. e. media with a symmetry group $G=O(g) \subset G L(T)$, where $T$ is a Euclidean vector space with a metric $g,(x, y) \stackrel{\text { def }}{=} g(x, y)$.

To this end we study, $O(g)$-orbits and $O(g)$-invariants of the natural $O(g)$ action on $\operatorname{End} T$ (see [10]).

Let $A \in \operatorname{End} T$ be a linear operator and $A^{\prime} \in \operatorname{End} T$ its adjoint operator with respect to the metric $g$.

Theorem 1 (Procesi [10]). Algebra of polynomial $O(g)$-invariants on $A \in$ End $T$ are generated by the Artin-Procesi invariants

$$
\mathcal{P}_{\alpha, \beta}(A)=\operatorname{Tr}\left(A^{\alpha_{1}} A^{\prime \beta_{1}} \cdots A^{\alpha_{m}} A^{\prime \beta_{m}}\right), \quad \sum_{i}\left(\alpha_{i}+\beta_{i}\right) \leq 2^{n}-1,
$$

where $\alpha=\left(\alpha_{1}, \ldots, \alpha_{m}\right), \beta=\left(\beta_{1}, \ldots, \beta_{m}\right)$ are multi-indices.

The next theorem follows from the Procesi theorem, the Rosenlicht theorem [9] and the observation that codimension of regular orbits equals

$$
\nu=n^{2}-\frac{n(n-1)}{2}=\frac{n(n+1)}{2} .
$$

Theorem 2. Field of rational invariant of the $O(g)$-action on $\operatorname{End} T$ is generated by any $\nu$ algebraically independent Artin-Procesi invariants. This field separates regular orbits.

Note that the Helmholtz free energy for Newtonian media is $O(g)$-invariant because of the trivial $O(g)$-action on $(s, \varepsilon, \theta)$, and therefore, due to the Rosenlicht theorem, $h=h\left(\theta, \mathcal{P}_{\alpha, \beta}(\Delta)\right)$ if $h$ is rational. 
In this case, due to (3), we have the following state equation:

$$
\sigma=\frac{\partial h}{\partial \Delta}=\sum_{\alpha, \beta} \frac{\partial h}{\partial \mathcal{P}_{\alpha, \beta}} \frac{\partial \mathcal{P}_{\alpha, \beta}}{\partial \Delta} .
$$

If we consider media, which satisfy 'Hooke's law', the Helmholtz free energy is a quadratic function of $\Delta$ and, therefore, has the form:

$$
h=\frac{1}{2}\left(a(\theta) \mathcal{P}_{2}(\Delta)+b(\theta) \mathcal{P}_{11}(\Delta)+c(\theta) \mathcal{P}_{1}^{2}(\Delta)\right)+d(\theta) \mathcal{P}_{1}(\Delta)
$$

where $a, b, c, d$ are some functions.

In this case the state equations take the form:

$$
\sigma=a(\theta) \Delta^{\prime}+b(\theta) \Delta+(c(\theta) \operatorname{Tr} \Delta+d(\theta)) \mathbf{1} .
$$

We call the functions $a, b, c$ viscosities, and $-d$ pressure, though they are not completely adequate to their names.

Usually, the cases when the operator $\Delta$ is self-adjoint are considered, in such cases there are only two viscosities.

\section{Thermodynamic invariants of Newtonian media with inner structure}

Let a Euclidean vector space $(T, g)$ be the orthogonal direct sum of a vertical $\left(V, g_{F}\right)$ and a horizontal $\left(H, g_{B}\right)$ Euclidean spaces, that is

$$
(T, g)=\left(V, g_{F}\right) \oplus\left(H, g_{B}\right)
$$

where $\operatorname{dim} V=m, \operatorname{dim} H=n$.

In this section we study invariants of linear operators End $T$ equipped with the natural $O\left(g_{F}\right) \times O\left(g_{B}\right)$-action.

Let $\Pi_{V}$ be orthogonal projector on $V$.

Theorem 3. Algebra of polynomial $O\left(g_{F}\right) \times O\left(g_{B}\right)$-invariants on $A \in \operatorname{End} T$ is generated by Artin-Procesi invariants

$$
\begin{gathered}
\mathcal{P}_{\alpha, \epsilon, \beta}(A)=\operatorname{Tr}\left(A^{\alpha_{1}} \Pi_{V}^{\epsilon_{1}} A^{\prime \beta_{1}} \cdots A^{\alpha_{k}} \Pi_{V}^{\epsilon_{k}} A^{\prime \beta_{k}}\right), \quad \sum_{i}\left(\alpha_{i}+\epsilon_{i}+\beta_{i}\right) \leq 2^{n+m}-1, \\
\text { where } \alpha=\left(\alpha_{1}, \ldots, \alpha_{m}\right), \epsilon=\left(\epsilon_{1}, \ldots, \epsilon_{m}\right), \beta=\left(\beta_{1}, \ldots, \beta_{m}\right) \text { are multi-indices. }
\end{gathered}
$$


It follows from the Rosenlicht theorem that we should expect the same number of generators in the field of rational $O\left(g_{F}\right) \times O\left(g_{B}\right)$-invariants, then dimension of a general orbit equals

$$
(n+m)^{2}-\frac{n(n-1)}{2}-\frac{m(m-1)}{2}=\frac{n(n+1)}{2}+\frac{m(m+1)}{2}+2 m n .
$$

Similar to the ordinary Newtonian media, for the Newtonian media with inner structure, we have the following state equations:

$$
\frac{\partial h}{\partial \Delta}=\sum_{\alpha, \epsilon, \beta} \frac{\partial h}{\partial \mathcal{P}_{\alpha, \epsilon, \beta}} \frac{\partial \mathcal{P}_{\alpha, \epsilon, \beta}}{\partial \Delta} .
$$

In the case when the media satisfy 'Hooke's law', the Helmholtz free energy is a quadratic function of $\Delta$ and therefore has the form:

$$
\begin{array}{r}
h=\frac{1}{2}\left(a_{1}(\theta) \operatorname{Tr} \Delta^{2}+a_{2}(\theta) \operatorname{Tr}\left(\Delta \Delta^{\prime}\right)+a_{3}(\theta) \operatorname{Tr}^{2} \Delta+a_{4}(\theta) \operatorname{Tr}^{2}\left(\Delta \Pi_{V}\right)+\right. \\
\left.a_{5}(\theta) \operatorname{Tr}\left(\Delta^{\prime} \Delta \Pi_{V}\right)+a_{6}(\theta) \operatorname{Tr}\left(\Delta \Delta^{\prime} \Pi_{V}\right)\right)+b_{1}(\theta) \operatorname{Tr} \Delta+b_{2}(\theta) \operatorname{Tr} \Delta \Pi_{V},
\end{array}
$$

where $a_{1}, \ldots, a_{6}, b_{1}, b_{2}$ are some functions.

In this case the state equations take the form:

$$
\begin{aligned}
\sigma= & a_{1}(\theta) \Delta^{\prime}+a_{2}(\theta) \Delta+\left(a_{3}(\theta)(\operatorname{Tr} \Delta)+b_{1}(\theta)\right) \mathbf{1}+ \\
& \left(a_{4}(\theta) \operatorname{Tr}\left(\Delta \Pi_{V}\right)+b_{2}(\theta)\right) \Pi_{V}+a_{5}(\theta) \Delta \Pi_{V}+a_{6}(\theta) \Pi_{V} \Delta .
\end{aligned}
$$

\section{Conservation laws and motion of media}

\subsection{Preliminaries}

The general laws of conservation of mass, momentum and energy are used to describe the motion of media. This can be done on an arbitrary Riemannian manifold $(M, g)$, the only difference between media with or without inner structure is in the description of a flow velocity field $X$ and the thermodynamics of media. Thus, for media without inner structure we have no extra conditions for vector fields and thermodynamics but in presence of inner structures we have to specify them.

Thus, in this section we consider a Riemannian manifold $(M, g)$ and write down the conservation laws for an arbitrary vector field $X$ on $M$. We'll assume that $M$ is an oriented manifold and $\Omega=\Omega_{g}$ is the volume form associated with the metric $g$. We denote by $\nabla$ and $d_{\nabla}$ the Levi-Civita connection and the covariant differential also associated with metric $g$. 
By

$$
\frac{d}{d t}=\frac{\partial}{\partial t}+\nabla_{X}
$$

we denote the material derivative.

\subsection{Divergence operator}

The divergence of a vector field, $\operatorname{div} X$, is defined by the standard way through Lie derivative:

$$
\mathcal{L}_{X} \Omega=(\operatorname{div} X) \Omega
$$

On the other hand, the covariant differential $d_{\nabla} X \in T^{*} \otimes T$ is a field of linear operators acting in the tangent spaces and we get

$$
\operatorname{div} X=\operatorname{Tr}\left(d_{\nabla} X\right)
$$

To see that we get an equivalent construction, let us write down the latter in local coordinates $x_{1}, \ldots, x_{n}$.

We get

$$
d_{\nabla} X=\sum_{i, j}\left(\frac{\partial X_{i}}{\partial x_{j}}+\sum_{k} \Gamma_{k j}^{i} X_{k}\right) \frac{\partial}{\partial x_{i}} \otimes d x_{j},
$$

where $X=\sum X_{i} \frac{\partial}{\partial x_{i}}$, and $\Gamma_{k j}^{i}$ are the Christoffel symbols.

Taking now $\left(x_{1}, \ldots, x_{n}\right)$ to be the normal coordinates we get equality (5).

This observation allows us to extend the divergence operator on other tensors. The more important case for us is the case of linear operators $A \in$ End $T=T \otimes T^{*}$.

In this case, $d_{\nabla} A \in T^{*} \otimes$ End $T=T^{*} \otimes T \otimes T^{*}$ and, by taking $(1,2)$ contraction $c_{1,2}$, we get a differential 1 -form that we call divergence of operator $A$ :

$$
\operatorname{div} A=c_{1,2}\left(d_{\nabla} A\right) \in T^{*} .
$$

In local coordinates we have

$$
\begin{aligned}
& A=\sum a_{i}^{k} \frac{\partial}{\partial x_{i}} \otimes d x_{k} \\
& d_{\nabla}\left(\frac{\partial}{\partial x_{i}}\right)=\sum \Gamma_{i j}^{k} \frac{\partial}{\partial x_{k}} \otimes d x_{j}, \\
& d_{\nabla}\left(d x_{i}\right)=-\sum \Gamma_{j k}^{i} d x_{k} \otimes d x_{j}
\end{aligned}
$$

and, therefore,

$$
\operatorname{div} A=\sum_{i, k}\left(\frac{\partial a_{i}^{k}}{\partial x_{i}}+\sum_{j}\left(a_{j}^{k} \Gamma_{i j}^{i}-a_{i}^{j} \Gamma_{i k}^{j}\right)\right) d x_{k} .
$$


Let $A=X \otimes \omega$, where $X$ is a vector field and $\omega$ is a differential 1-form. Then it is easy to check that

$$
\operatorname{div}(X \otimes \omega)=(\operatorname{div} X) \omega+\nabla_{X} \omega .
$$

We consider the stress tensor $\sigma \in \operatorname{End} T$ as the surface force $\widehat{\sigma}=g(\sigma(\nu), \cdot)$ $\in T^{*}$ applied to an imaginary surface orthogonal to a normal vector $\nu$. In our case we cannot directly find the 'integral sum' of all forces applied to a volume, since each of the 'applied forces' belongs to a different space.

Take a small volume $\Delta V$ with a border $S$ around a center point $a \in M$ and consider parallel transports $\gamma_{a, x}: T_{a} \rightarrow T_{x}$ along the shortest geodesic line connected points $x$ and $a$.

Let $f(x)=\gamma_{a, x}^{*}(\widehat{\sigma}) \in T_{a}^{*}$ be the images of the surface forces. Then the force applied to the volume we'll be understand the integral $\int_{S} f d s$, where $f$ considered as a vector valued function $f: \Delta V \rightarrow T_{a}^{*}$

To see that the density of this force equals $\operatorname{div} \sigma$ let take normal coordinates $\left(x_{1}, \ldots, x_{n}\right)$ centered at the point $a$. Then $g_{i j}=\delta_{i j}+o_{2}(x), \Gamma_{i j}^{k} \in o_{1}(x)$, where we denoted by $o_{l}(x)$ functions having zero of order $l$ at the point $a$, and therefore, due to the above formula, $\operatorname{div} \sigma=\sum \sigma_{i, i}^{k}(a) d x_{k}$ at the point $a$.

On the other hand, $f(x)=\sum\left(\sigma_{i}^{k}(x)+o_{1}(x)\right) \nu_{i}$ and, therefore,

$$
\int_{S} f d s=\int_{S} \sum \sigma_{i}^{k}(x) \nu_{i} d s+o_{1}(\Delta V)=\left(\operatorname{div}_{a} \sigma\right) \Delta V+o_{1}(\Delta V),
$$

i. e. the density of internal force is $-\operatorname{div} \sigma$.

\subsubsection{Conservation of mass}

The idea that the amount of a medium contained in an elementary volume is conserved along the flow of the medium can be expressed with the equation

$$
\left(\frac{\partial}{\partial t}+\mathcal{L}_{X}\right)(\rho \Omega)=0
$$

or equally

$$
\frac{d \rho}{d t}+\rho \operatorname{div} X=0
$$

\subsubsection{Conservation of momentum, or the Navier-Stokes equation of motion}

Considering the metric $g$ as the isomorphism $g: T \rightarrow T^{*}$, denote by $X^{b}=$ $g(X) \in T^{*}$ the differential 1 -form dual to a vector field $X$, and by $\alpha^{b}$ the vector field dual to a differential 1-form $\alpha$. 
Then a differential form $\omega=\rho X^{b}$ may be considered as the momentum density of the media.

Newton's second law states that the force equals to the mass multiplied by the acceleration. In our case, $\frac{d X}{d t}$ is the acceleration and, therefore, the law states that

$$
\rho \frac{d X}{d t}=-\operatorname{div}^{b} \sigma
$$

The Levi-Civita connection preserves the metric $g$. Therefore, due to the above formula, we have

$$
\rho \frac{d X^{b}}{d t}=-\operatorname{div} \sigma
$$

and due to (6) we get

$$
\frac{\partial \omega}{\partial t}+\operatorname{div}(X \otimes \omega)=-\operatorname{div} \sigma
$$

The last relation in the form

$$
\frac{\partial \omega}{\partial t}+\operatorname{div}(X \otimes \omega+\sigma)=0
$$

is called the equation of the momentum conservation.

\subsubsection{Conservation of energy}

The law of energy conservation is written as follows

$$
\frac{\partial e}{\partial t}=-\operatorname{div} \mathcal{J}_{e}
$$

where $\mathcal{J}_{e}$ is the total energy flux vector.

This vector is the sum of a convective term $e X$, a mechanical energy flux $\sigma(X)$ and a heat flow $\mathcal{J}_{q}$ (for details, see [15]):

$$
\mathcal{J}_{e}=e X+\sigma(X) \mathcal{J}_{q}
$$

Substitution the velocity field $X$ into (8) leads to the kinetic energy balance equation

$$
\rho \frac{d}{d t} \frac{(X, X)}{2}=-(\operatorname{div} \sigma)(X)+(\sigma, \Delta)
$$

Recall that the total energy enclosed in an elementary volume is the sum of kinetic, potential and internal energy

$$
e=\frac{\rho\langle X, X\rangle}{2}+\varepsilon
$$


Using this identity and the equations (7), (11) we get

$$
\frac{d}{d t} \varepsilon+\varepsilon \operatorname{div} X+\operatorname{div}\left(\mathcal{J}_{q}\right)+\langle\sigma, \Delta\rangle=0 .
$$

Commonly, the heat flow is given by Fourier's law

$$
\mathcal{J}_{q}=-\chi(\operatorname{grad} T),
$$

where $\chi \in \operatorname{End} T$ is the thermal conductivity of the medium.

Summarizing we have the following system of differential equations describing media with inner structures:

$$
\left\{\begin{array}{l}
\frac{d \rho}{d t}+\rho \operatorname{div} X=0 \\
\rho \frac{d X}{d t}=-\operatorname{div}^{b} \sigma \\
\frac{d \varepsilon}{d t}+\varepsilon \operatorname{div} X+\operatorname{div}\left(\mathcal{J}_{q}\right)+\langle\sigma, \Delta\rangle=0
\end{array}\right.
$$

where

$$
\sigma=\frac{\partial h}{\partial \Delta}, \quad \varepsilon=h+\theta \frac{\partial h}{\partial \theta},
$$

$X$ is a $\pi$-projectable vector field.

\section{Acknowledgements}

All three authors are partially supported by the Russian Foundation for Basic Research, Grant 18-29-10013.

Valentin Lychagin and Sergey Tychkov are partially supported by the Foundation for the Advancement of Theoretical Physics and Mathematics "BASIS", Grant 19-7-1-13-2.

\section{References}

[1] Batchelor, G. K. An introduction to fluid dynamics. Cambridge Mathematical Library. Cambridge University Press, Cambridge, 1999. xviii+615 pp.

[2] Gorinov, Anton A.; Lychagin, Valentin V.; Roop, Mikhail D.; Tychkov, Sergey N. Gas flow with phase transitions: thermodynamics and the Navier-Stokes equations. Nonlinear PDEs, their geometry, and applications, 209-222, Tutor. Sch. Workshops Math. Sci., Springer, 2019 
[3] Duyunova, A. A.; Lychagin, V. V.; Tychkov, S. N. Classification of the thermodynamic state equations of a viscous fluid. (Russian) Dokl. Akad. Nauk 473 (2017), no. 6, 635-639; translation in Dokl. Math. 95 (2017), no. $2,172-175$

[4] Duyunova, Anna; Lychagin, Valentin; Tychkov, Sergey Differential invariants for spherical layer flows of viscid fluids. J. Geom. Phys. 130 (2018), 288-292.

[5] Kruglikov Boris, Topological classification of Leggett systems in an integrable case for 3He-A, Russ. Math. Surv., 46 (4), pp. 179-181,1991.

[6] Kruglikov Boris, Lychagin Valentin, Global Lie-Tresse theorem, Selecta Math. (NS) 22 (3) (2016) 1357-1411.

[7] Lychagin, Valentin, Contact geometry, measurement, and thermodynamics. Nonlinear PDEs, their geometry, and applications, 3-52, Tutor. Sch. Workshops Math. Sci., Springer, 2019.

[8] Lychagin, Valentin; Roop, Mikhail; Real gas flows issued from a source. Anal. Math. Phys. 10 (2020), no. 1.

[9] Rosenlicht M., A remark on quotient spaces, An. Acad. Brasil. Cienc. 35 (1963) 487-489.

[10] Procesi C., Lie groups: an approach through invariants and representations, Springer, (2005).

[11] Vardoulakis, Ioannis. Cosserat Continuum Mechanics: With Applications to Granular Media. Vol. 87. Springer, 2018.

[12] Cosserat, Eugene, and François Cosserat. Thèorie des corps déformables. (1909).

[13] Spencer A. J. M.; Rivlin R. S. Further results in the theory of matrix polynomials. Arch. Rational Mech. Anal. 4 (1960), 214-230.

[14] Spencer, A. J. M. Theory Invariants, in Continuum Mechanics. vol.1, Longman, (1980).

[15] De Groot, Sybren Ruurds, and Peter Mazur. "Non-equilibrium thermodynamics." (2013). 